ARCHIVO ESPAÑOL DE ARTE, LXXX, 320

OCTUBRE-DICIEMBRE 2007, pp. 367-377

ISSN: 0004-0428

\title{
DOS IMPORTANTES PINTURAS FLAMENCAS INÉDITAS ATRIBUIDAS AL MAESTRO DE FRANKFURT Y A PETRUS CHRISTUS
}

\author{
POR \\ ELISA BERMEJO \\ Investigadora-CSIC
}

\begin{abstract}
Se estudia una nueva obra del Maestro de Frankfurt definiendo una serie de caracteres que permiten establecer una serie de conclusiones sobre la personalidad del artista, uno de los pintores más avanzados de su época. Se completan las noticias que se dieron sobre el Tríptico de El Salvador de la Catedral de Ávila que atribuimos a Petrus Christus.
\end{abstract}

Palabras clave: Maestro de Frankfurt. Petrus Christus. Tríptico de El Salvador. Catedral de Ávila.

The author studies a new work by the Frankfurt Master, defining a series of characteristics which allow for the establishment of conclusions concerning the personality of the artist, one of the most advanced painters of his time. Further information is given on the Triptych of our Saviour in Avila Cathedral, here attributed to Petrus Christus.

Key words: Frankfurt Master. Petrus Christus. Triptych of our Saviour. Avila Cathedral.

Hace unos veinticinco años tuve la ocasión de conocer una espléndida y curiosa pintura que, en esa fecha, era propiedad privada de un coleccionista catalán domiciliado en Barcelona y que actualmente se conserva en colección particular de Cantabria. Se trata de un óleo sobre tabla de roble que mide 115 x $80 \mathrm{~cm}$ y representa La Presentación del Niño Jesús en el Templo. Ya entonces me dio una impresión de gran interés por varios y curiosos detalles (figs. 1-5).

Lo que más me llamó la atención fue el ángulo inferior derecho de la pintura en el que aparecen dos figuras que se supone representan a los Donantes de la obra.

Desconocemos la fecha de la llegada de la pintura a la colección catalana pero el dueño me comentó, verbalmente, que todo ese pequeño espacio estuvo, en un tiempo, pintado de negro, noticia sin dato seguro que la pudiese justificar.

Para llegar a un conocimiento lo más acertado posible creemos conveniente hacer un detenido estudio del conjunto de esta interesante obra que por sus caracteres estéticos y la calidad de las figuras y elementos que dan color y alegría a la escena, recuerda el estilo del pintor conocido como Maestro de Frankfurt del que, por el momento, se tienen muy pocos datos que puedan 


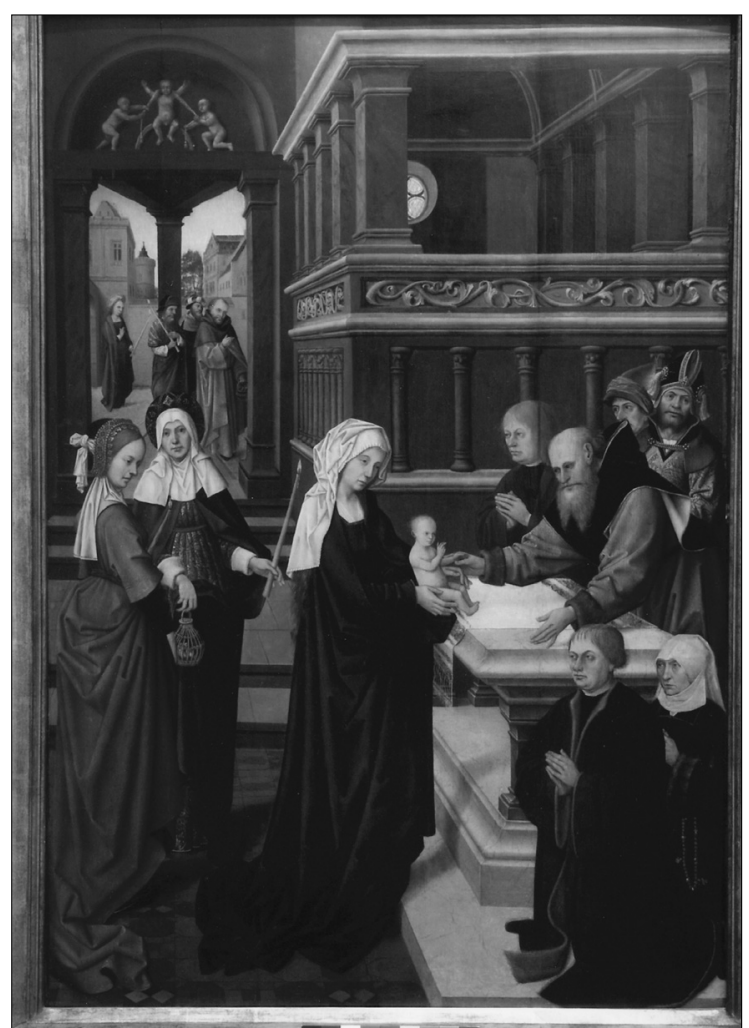

Fig. 1. Maestro de Frankfurt. La Presentación del Niño Jesús en el Templo. Santander. Colección particular.

individualizarle con seguridad. Nos conviene buscar detalles que, comparados con otros que aparecen en obras que se vienen atribuyendo a este artista, nos sirvan para considerar la pintura como una nueva obra de este pintor en España.

En primer lugar cabe destacar la disposición de las escenas y la situación de las figuras que la componen. En la mitad del espacio vertical se instalan las figuras principales que configuran el grupo que representa la Presentación del Niño en el Templo Aparece como personaje fundamental la Virgen, sosteniendo en sus brazos a Jesús. Esta figura está diseñada con un aspecto de elegancia y finura que el pintor ha cuidado de una forma exquisita, con el manto azul deslizándose suavemente desde los hombros hasta el suelo.

Tras ella aparecen dos bellas jóvenes que la acompañan, una de las cuales lleva la cesta de las dos tortolitas que suele acompañar a la representación de este tema de la Presentación del Niño. Precisamente estos dos personajes aportan a la escena una sensación fresca y alegre en sus rostros y actitudes.

En la parte de la derecha esta colocada la mesa-altar donde la Virgen se ve dispuesta a entregar el Niño al Sacerdote. Es original y de gran efecto la actitud de éste, que abre sus brazos dispuesto a recoger tan importante presente. También nos llama la atención los personajes que le acompañan y que por sus rostros dan la impresión de ser figuras tomadas del natural.

Muy curioso nos resulta el elemento arquitectónico que parece querer representar un deseo italianizante pero que, como es conocido en las pinturas de los artistas nórdicos, lo convierten en un recuerdo y ello se ve, aún más claro, en el relieve escultórico representado en el tímpano del arco que da paso al exterior y por el que hace su entrada San José para asistir al solemne acto que va a tener lugar. Los ángeles representados en dicho relieve responden a un claro estilo italiano. 


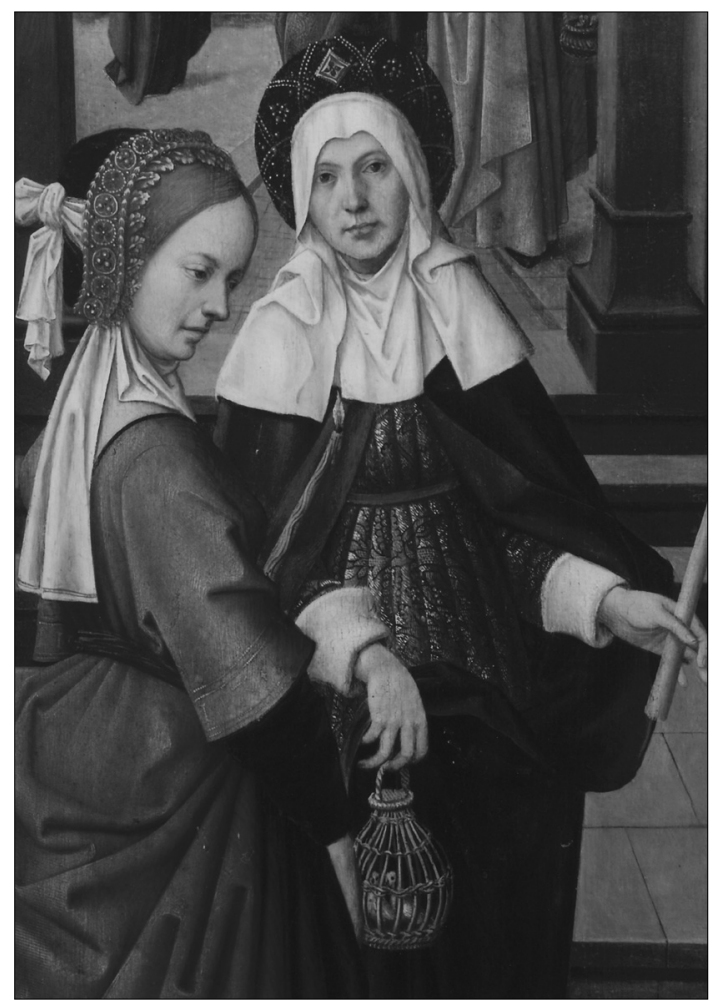

Fig. 2. Detalle de la fig. 1.

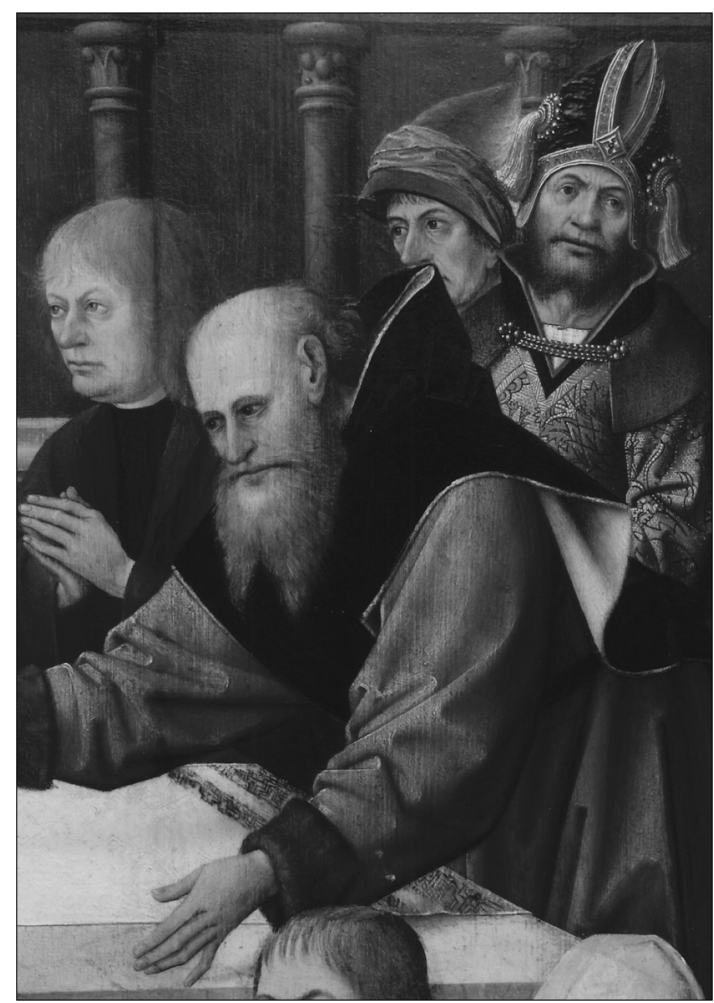

Fig. 3. Detalle de la fig. 1.

Hemos presentado una visión general del cuadro para destacar los detalles más interesantes, sobre todo con la intención de buscar algunos elementos que puedan acercarnos a captar, quizás, algún dato para ayudarnos a conseguir el posible nombre de un pintor tan interesante.

Quizás lo más llamativo es la representación de los que aparecen como Donantes de la obra que, como hemos apuntado al inicio y conocemos por declaración sólo verbal del anterior propietario, ocupan el pequeño espacio que en tiempos estuvo cubierto de pintura negra.

Hemos tenido la suerte de que un amigo del actual propietario que no quiere dar a conocer su identidad, se brindó a hacer una revisión de la pintura y con experto conocimiento nos dio la alegría de confirmar que este detalle de los Donantes pertenece a la pintura original. El resultado de la prueba a la que sometió la tabla trascrito literalmente fue el siguiente: Se ha realizado una exploración de la totalidad de la superficie de la tabla habiéndose encontrado fluorescencias en la parte baja del altar próxima a los oferentes; igualmente se han encontrado fluorescencias en la toca de color blanco de la Oferente. En el resto de la pintura no se aprecia ninguna otra fluorescencia. Adjuntamos fotografía en la que se señalan las zonas donde se han encontrado las fluorescencias ${ }^{1}$.

Por mi parte hemos estudiado las figuras que representan a los Donantes, comparándolas con el que se considera autorretrato del Maestro de Frankfurt con su mujer en colección privada suiza (fig. 6) y creemos encontrar cierto parecido con los rostros de los personajes de una y otra pintura. Lo más

\footnotetext{
${ }^{1}$ Prueba a la luz ultravioleta para la localización de posibles pigmentos de pintura con bióxido de Titanio (Rutilio) en tabla flamenca, siglo XVI, atribuida al Maestro de Frankfurt. Equipo utilizado: Marca P.A.C.I.S.A. (Madrid-Barcelona) Equipo de luz ultravioleta, con lámpara OSRAM (Made in Germany) tipo HQV, potencia 125 W p.d.A., corriente 125 V.C.A...
} 
sobresaliente, pensamos, es la anchura de las aletas de la nariz del caballero. El rostro fino de la joven esposa también se asemeja al de la Donante, así como su estatura.

Otros temas interesantes para acercarnos un poco más al Maestro de Frankfurt, es la afición por introducir elementos con diseño de brocado que aparecen en la indumentaria de buena parte de los personajes, en sus vestiduras y tocados, incluso en una de las jóvenes que aparecen tras la Virgen figura un brocado que representa en dorado las letras "I", mayúscula, "C", mayúscula y el signo “ “, que podría ser otro dato para reconocer el nombre del pintor. Pudieran ser las iniciales del nombre o, lo que es más probable, marca del artista que se encargó de realizar los brocados. Dado el gusto por estos detalles que el artista demuestra con más interés y abundancia en sus pinturas más avanzadas pudiera también ser una indicación de haber añadido a la profesión de pintor la del ejercicio del brocado.

Como puede verse en la fotografía del detalle de los personajes que acompañan al Oficiante, se aprecia en sus tocados una abundante decoración que añade a la pintura una afición muy personal por el brocado por lo que quizás cabe suponer que el Maestro de Frankfurt hubiera reunido ambas profesiones.

Hay que destacar otros detalles menos evidentes para un posible reconocimiento de su identidad pero que dan una idea de la elegancia y sensibilidad del pintor. Nos referimos al interés que presta a la forma de presentar la Virgen al Niño, con una figura blanquecina y luminosa que se refleja sobre la mesa-altar que sirve de espacio para entregarle al Oficiante y que produce un efecto de luz que llama la atención del espectador y atrae su visión a la importancia de la celebración.

También es una actitud interesante la idea de la entrada de San José por el vano que pone en comunicación los dos ambientes, el del Templo, con su atmósfera religiosa, con la luz y vitalidad del exterior.

Desafortunadamente no es posible conocer una fecha determinada para la noticia de la posible publicación de fotografías en color en nuestra Revista, pero la finura y elegancia del color que se percibe en la pintura, nos invita a hacer algunos comentarios que puedan aliviar la falta de medios necesarios pero no obtenidos, para la publicación que nos ocupa.

El pintor da muestra de un conocimiento pictórico de elegancia y virtuosismo, lo que le permite unir la expresión con el deseo de conseguir un avance en su evolución artística. Tiene, además, facilidad para situar los personajes de forma que expresen, de una parte, la importancia de cada uno de ellos y, al mismo tiempo, el avance que aportan. Así, en las dos figuras femeninas que aparecen tras la Virgen se percibe el deseo de añadir a la manifiesta elegancia de la representación, el gesto alegre y naturalista que se percibe en el rostro de las dos.

Otra nota interesante es el equilibrio de los propios colores, como puede advertirse en las figuras de la derecha donde hay una novedosa mezcla de tonos sin diferenciarlos, sino utilizando la mezcla de los más destacados. Esto es visible en la indumentaria del Oficiante Simeón en la que se combina el azul del manto de la Virgen con el tono rojizo que le da una visión armoniosa.

La austeridad de Simeón y el tono negro de la vestidura del personaje que está a su derecha queda contratada por el color y la elegancia decorativa que se percibe en los que quedan a su fondo, no solo por el color sino por la riqueza de sus adornos.

No hemos de olvidar, tampoco, el fondo del cuadro en el que se contrapone la tonalidad de la arquitectura y la escultura en sus tonos precisos que le permite dar una luminosa y alegre vitalidad de espacio abierto a la ciudad.

El estudio de las obras del Maestro de Frankfurt nos lleva a fechas muy tempranas. El gran altar de Santa Ana, cuyas tablas están ahora en el Museo de la ciudad, fue consagrado en $1492^{2}$.

\footnotetext{
${ }^{2}$ FrIEDLÄNDER, Max J. Early Netherlandish Painting. VII, 1971:129, láms. 100-101.- WeIZsÄCKER. Die Kunstschätze des Dominikar Klosters (Convento de Dominicas), Frankfurt, 127.
} 


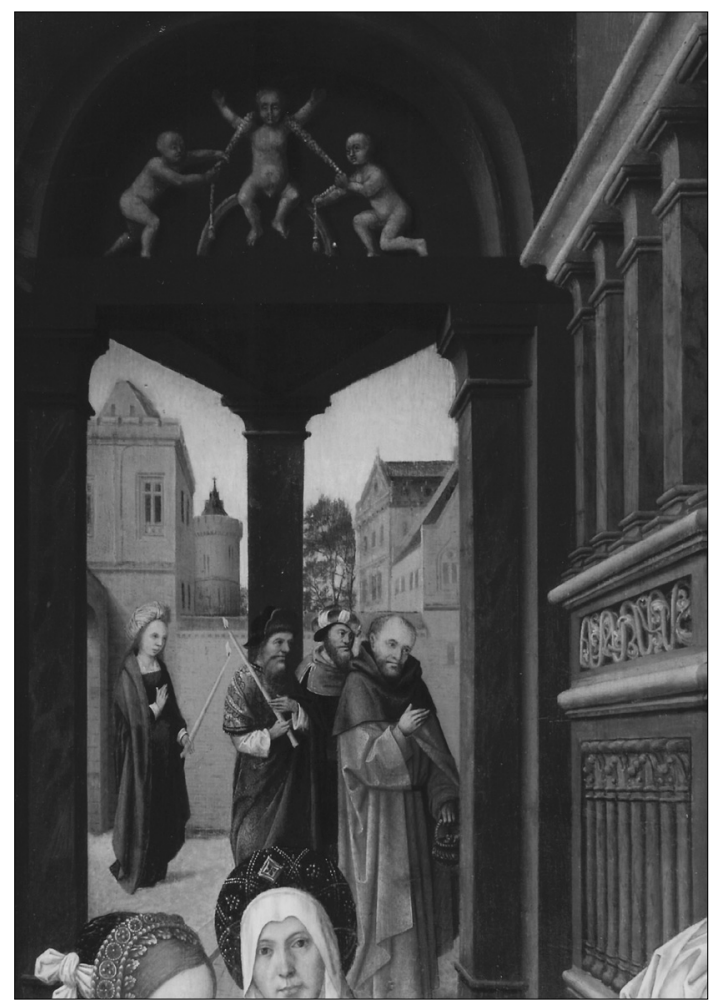

Fig. 4. Detalle de la fig. 1.

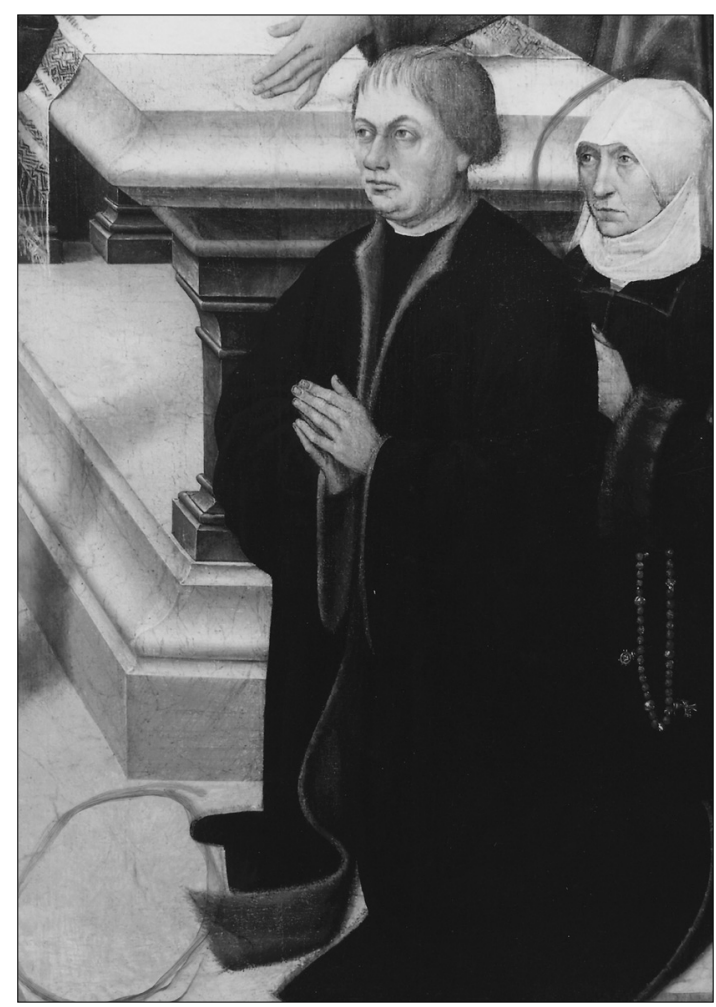

Fig. 5. Detalle de la fig. 1.

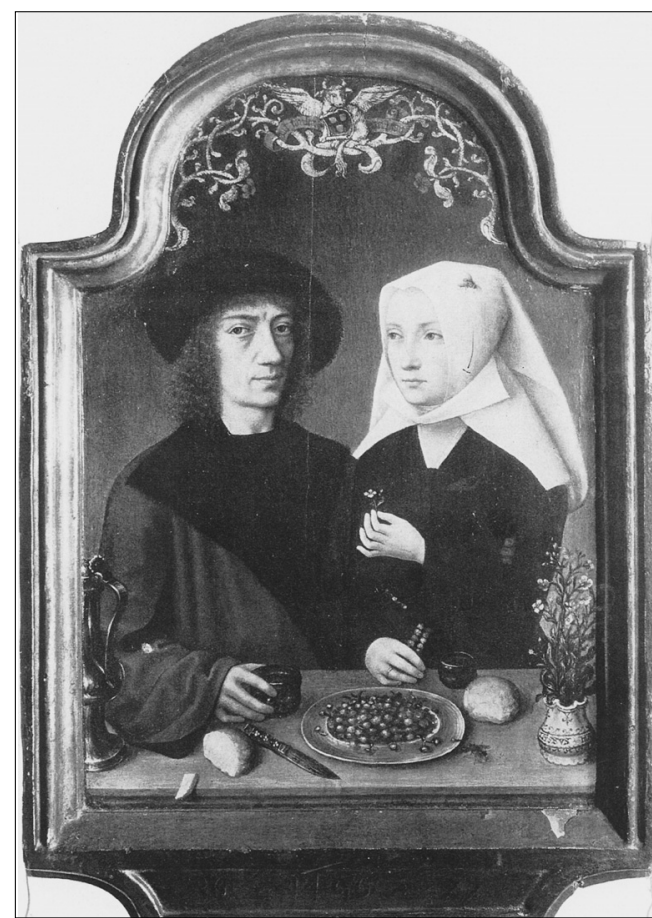

Fig. 6. Maestro de Frankfurt. Retrato doble del Maestro y su esposa. Ginebra. Colección particular. 
El Maestro de Frankfurt era mayor que Massys. Aunque no sabemos su nombre, obtenemos varios detalles biográficos de un retrato doble en el que se pintó a si mismo junto con su esposa 3 . Este retrato de gran significado para la historia del arte en Amberes, fue vendido por un coleccionista privado de Inglaterra a la Colección Von Auspitz, en Viena. Actualmente se encuentra en una colección privada en Ginebra 4 .

En la parte superior aparece la enseña del Gremio de Pintores de Amberes, dentro de una ornamentación y sostenido por el toro alado de San Lucas, con tres escudos suavemente coloreados junto con la inscripción In Jonstem Versamt - 1271 (Unidos en la amistad). Esta inscripción parece indicar con claridad que el artista pertenecía al grupo de pintores de Amberes.

En la parte inferior del marco original aparecen las cifras:36-1496-17. Claramente se refieren a un Maestro de Amberes de 36 años (nacido en 1460) con la esposa de 175 que aparece, seguramente justo después de su boda, sentada a la mesa, disfrutando feliz de su nuevo ambiente hogareño. El Maestro tiene un aspecto joven, enjuto, con una nariz de anchas aletas, cabello castaño cayendo sobre su frente que está dividida en dos por un entrecejo vertical y gruesas cejas.

Sobre el Maestro de Frankfurt puede decirse que toma su nombre del lugar en que se encuentras dos de sus importantes trípticos, el de la Crucifixión con Donantes, en el Stäldesches Kunstinstitut y La Familia de Santa Ana, en el Historisches Museum, ambos en Frankfurt.

Sánchez Cantón, en el Catálogo del Museo del Prado ${ }^{6}$ refiriéndos a la tabla de Santa Catalina, puerta izquierda de un tríptico, dice que procede del convento de Santa Cruz de Segovia. Según Friedländer es obra tardía del Maestro de Frankfurt y según Winkler obra de B. Van Orley. Junto con su tabla compañera de Santa Bárbara vinieron al Prado del Museo de la Trinidad.

La opinión más aceptable sobre su identificación proviene de Vanaise ${ }^{7}$ el cual reune un grupo de obras en relación estilística con los dos trípticos primeros citados, que prestan su nombre al pintor, y otro tríptico que parece de distinta mano y al que provisionalmente denomina Maestro de Wateruliet.

Se han propuesto también los nombres de Jan de Vos y de Heyndrik van Wuelwe, aunque de ellos sólo nos han llegado datos documentales sobre su existencia, pero no se conoce obra suya que pueda servir como referencia artística ${ }^{8}$. Nuestra opinión se esbozó en varios de nuestros trabajos ${ }^{9}$.

\section{El Tríptico de Cristo Salvador, obra de Petrus Christus (atribución), en la Catedral de Ávila}

En el Museo de Ávila, como Depósito de la Diputación provincial, se conserva el Tríptico de Cristo Salvador, sobre el que se dio noticia escueta hace algunos años con intención de apoyar con nuevos argumentos su atribución a Petrus Christus en posterior publicación, lo que con algún retraso emprendemos ahora ${ }^{10}$ (figs. 7 y 8 ).

\footnotetext{
3 FRIEDLÄNDER,VII, cit. 163, lám-117.

4 Según Stephen H. GodDard, el cuadro de encuentra actualmente en el Köninklijk Museum voor Schöne Kunsten, de Amberes."Brocade patterns in the shop of the Master of Frankfurt: an accessory to stylistic analysis" Art Bulletin, vol.LII, $\mathrm{n}^{\circ} 3$, sept. 1985.

5 GodDARD, cit. opina que la edad de la esposa es de 27 años.

${ }^{6}$ Madrid, $1972 . n^{\circ}$ s. 1941 y 1942.

7 Vanaise, P. "Le tryptique de la Déploration de l'eglise de Wateruliet", Bulletin de l'Institut Royale du Patrimoine Artístique, IX,1966.

8 ValentineR, W.R. "Jan de Vos, the Mester of Francfort", The Art Quaterly, VIII, 1945.

9 BermeJo, Elisa. La Pintura de los Primitivos flamencos en España”, 1980.- IDEM Tesoros de las Colecciones particulares madrileñas”Tablas españolas y flamencas, 1300-1550”, Catálogo de la Exposición. Real Academia de Bellas Artes de San Fernando, 1988, n 22. IDEM-Tablas Flamencas en la Ruta Jacobea, Exposición Itinerante. Oviedo, 1999, Santa Catalina y Santa Bárbara. p.100, fgs.64-65 y Trítptico de la Virgen co el Niño en un paisaje, p.246, 247,248.

10 Bermejo, Elisa. "Tríptico de Cristo Salvador", Testigos. Las Edades del Hombre. Ávila, 2004, 544-546.
} 


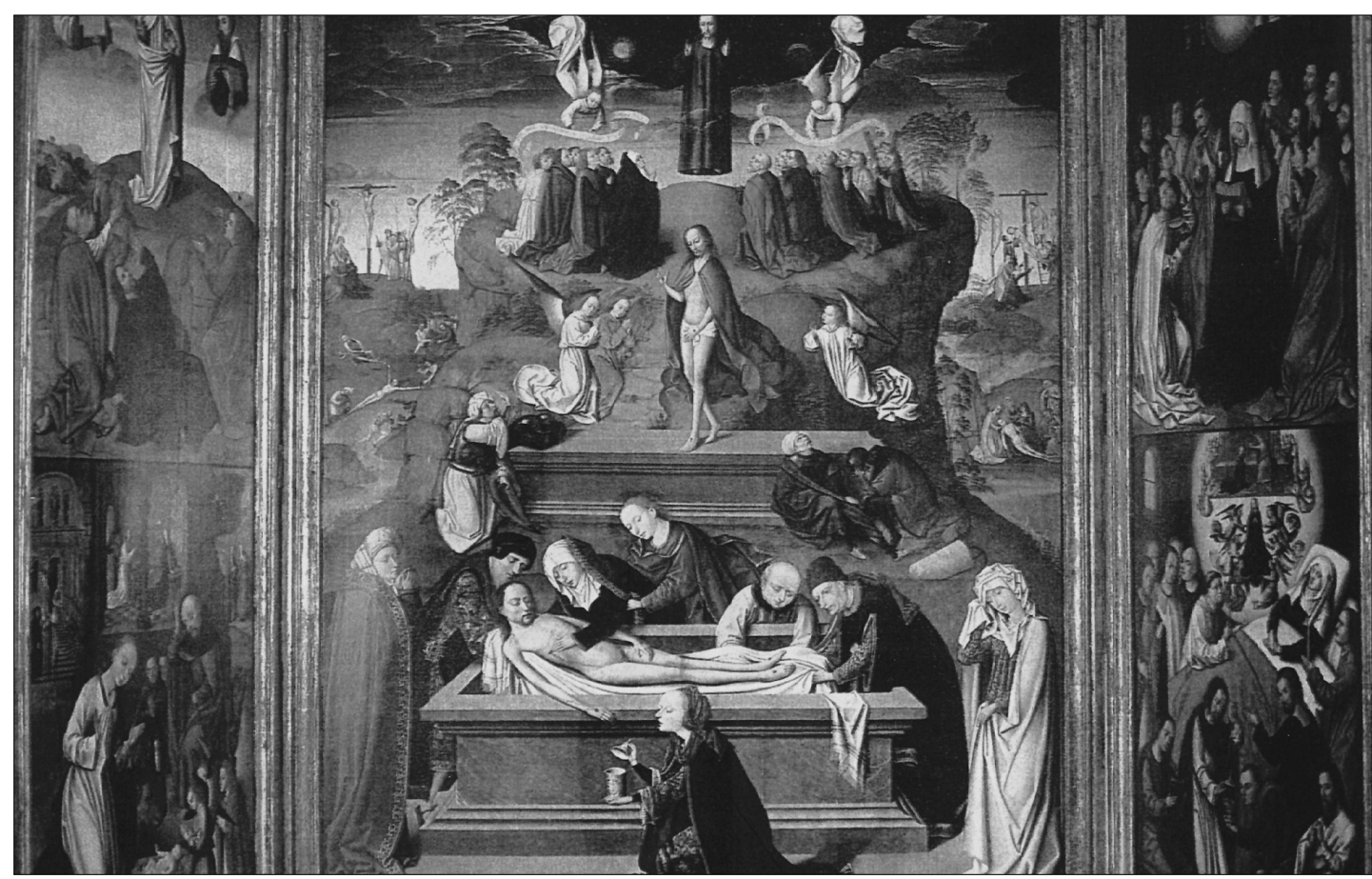

Fig. 7. Petrus Christus (atrib.). Tríptico del Cristo Salvador. Ávila. Museo (en depósito).

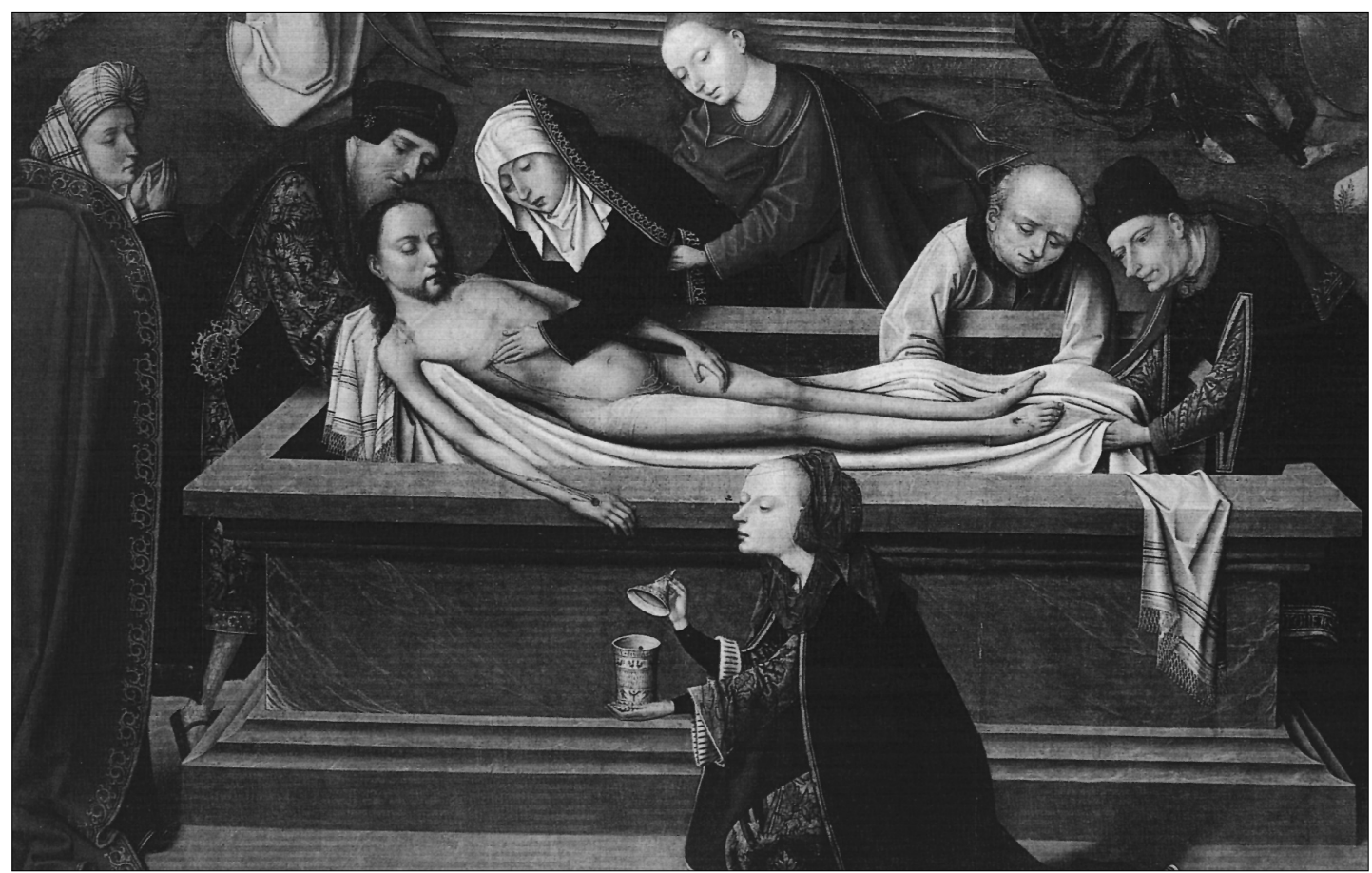

Fig. 8. Detalle de fig. 7.

AEA, LXXX, 320, OCTUBRE-DICIEMBRE 2007, 367-377, ISSN: 0004-0428 
El tríptico, sobre soporte de roble, mide 223x $345 \mathrm{~cm}$ En su tabla central, en el primer plano inferior, se representa el Entierro de Cristo, con las figuras de José de Arimatea y Nicodemo que, ayudados por un tercer personaje, depositan el cuerpo exánime de Cristo sobre un lienzo de inmaculada blancura. La imagen de la Virgen se inclina angustiada sobre la cabeza de Cristo, sostenida a medias por la figura de san Juan. La Magdalena, arrodillada delante del féretro, sostiene su tarro de perfumes mientras que otras dos compungidas figuras femeninas les contemplan. Sobre esta escena el Cristo Resucitado entre ángeles centra toda la composición de esta tabla central que en su parte superior reproduce la escena de la Ascensión con la figura del Salvador que se eleva a los cielos contemplado por las figuras de los Apóstoles y la Virgen arrodilladas y dispuestas en dos semicírculos.

En su puerta izquierda, en su recuadro inferior, se representa la Natividad sobre la que en pequeño sobre un fondo de tonos más apagados que acentúan los efectos de las perspectivas arquitectónicas, aparecen las escenas de la Anunciación y de la Presentación de María en el Templo. En el recuadro superior de esta portezuela aparece la Transfiguración en la que el fuerte colorido de la indumentaria de los tres apóstoles, san Pedro, san Juan y Santiago, contrasta con la claridad de un fondo de paisaje sobre el que destaca la figura erguida de Cristo entre Elías y Moisés de medio cuerpo.

La portezuela de la derecha representa en su recuadro inferior la Muerte de la Virgen, escena de un interior en la que se expone una serie abigarrada de personajes agrupados sabiamente sobre la que aparecen, en una especie de nimbo luminosos, la Asunción y la Coronación de María. En la parte superior la Venida del Espíritu Santo o Pentecostés en un interior de tonos oscuros sobre el que el resplandor del nimbo del Espíritu Santo juega un efecto claroscurista muy pronunciado.

Desde el punto de vista de su interno significado iconológico, la escena del Cristo en el sepulcro nos acerca al momento humano de la muerte sobre la que la figura del Resucitado y su Ascensión a los cielos plantea el núcleo central del Cristianismo. Las representaciones en las portezuelas son un recital conjunto de la vida de Cristo y la Virgen.

Desde el punto de vista artístico llama la atención la importancia y variedad que el autor, plásticamente y con un profundo sentido narrativo, presenta cada una de las escenas que ha seleccionado para poner ante el espectador todo un programa de meditación interna conduciendo su espíritu hacia un especial sentimiento interior de auténtica piedad ante la contemplación de Cristo como Salvador de la Humanidad, de acuerdo a la devoción de la época que preludia la Contrarreforma.

Al estudiar este importante Tríptico advertimos desde un primer momento la similitud estilística que presentaban sus escenas con la obra documentada de Petrus Christus. De este pintor, admitido como maestro en el Gremio de pintores de la ciudad de Brujas el 6 de julio de 1444, se ha dicho que se había descuidado el análisis de su obra debido a ser contemporáneo de Jean van Eyck, pintor oficial de la Corte, establecido en esta misma ciudad de Brujas, el cual acaparaba todos los encargos más importantes de su época.

Los escasos datos que se conocen sobre la vida de Petrus Christus dan a conocer que estuvo casado y que tuvo un hijo llamado Bastín que fue miniaturista. Sus obras firmadas y fechadas se escalonan entre los años de 1446 y 1457 y su estudio ha permitido adjudicarle un extenso número de obras de su mismo estilo. Se calcula que trabajó durante cerca de treinta años dedicándose a encargos de retratos y pintura devocional, en general trípticos de altar, conociéndose que se le encargó un monumental Arbol de Jessé para una procesión dedicada a una Santa. Su obra conservada ha permitido la comparación del tríptico abulense con su producción pictórica que justificase nuestra atribución.

Sin extendernos demasiado en nuestras apreciaciones solo queremos destacar la semejanza de la escena inferior del Entierro de Cristo con la que aparece en la tabla de La Lamentación conservada en el Metropolitan Museum de Nueva York (fig. 9) que Friedländer consideraba obra de Petrus 


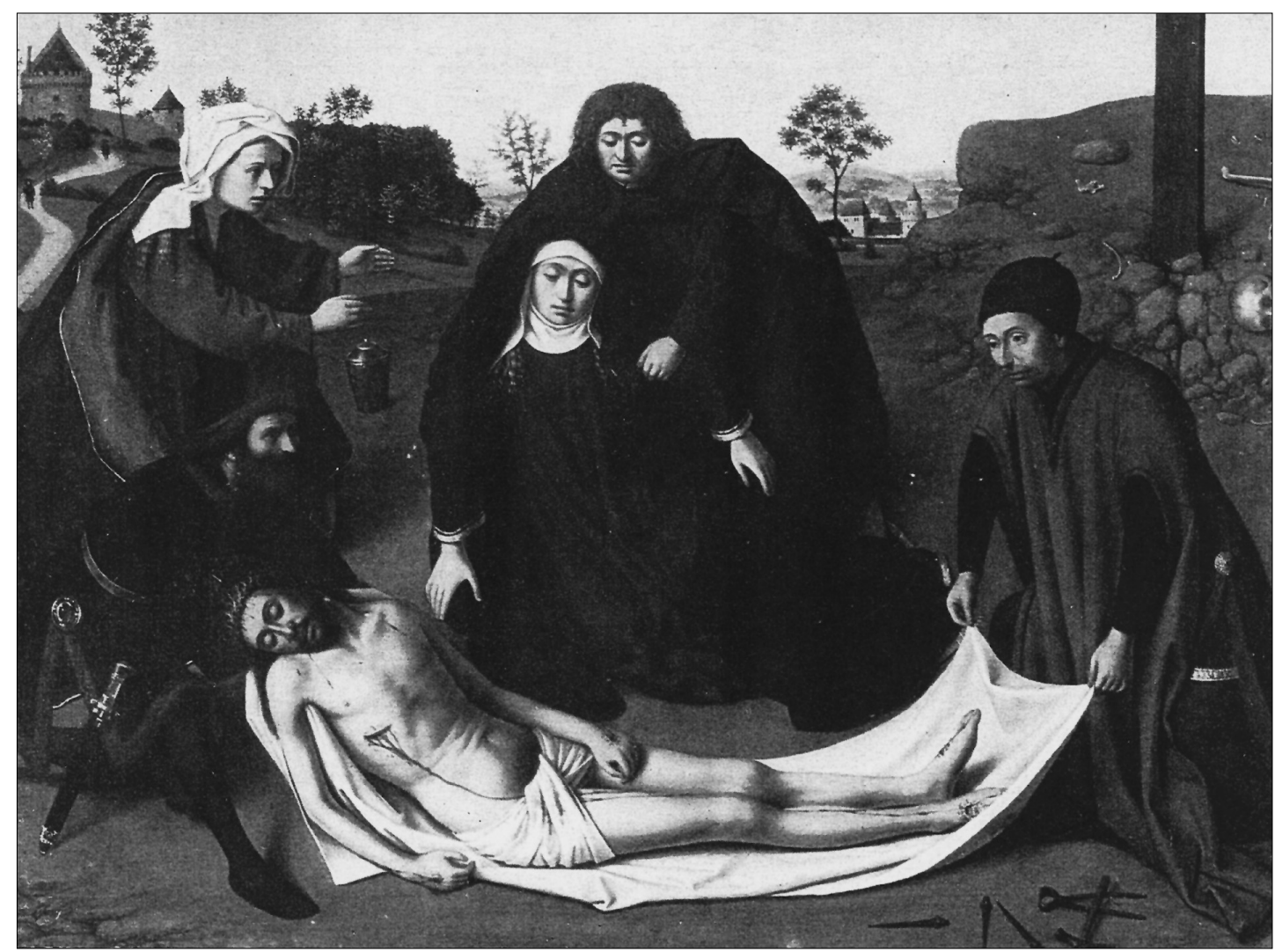

Fig. 9. Petrus Christus. La Lamentación. Nueva York Metropolitan Museum. of Art.

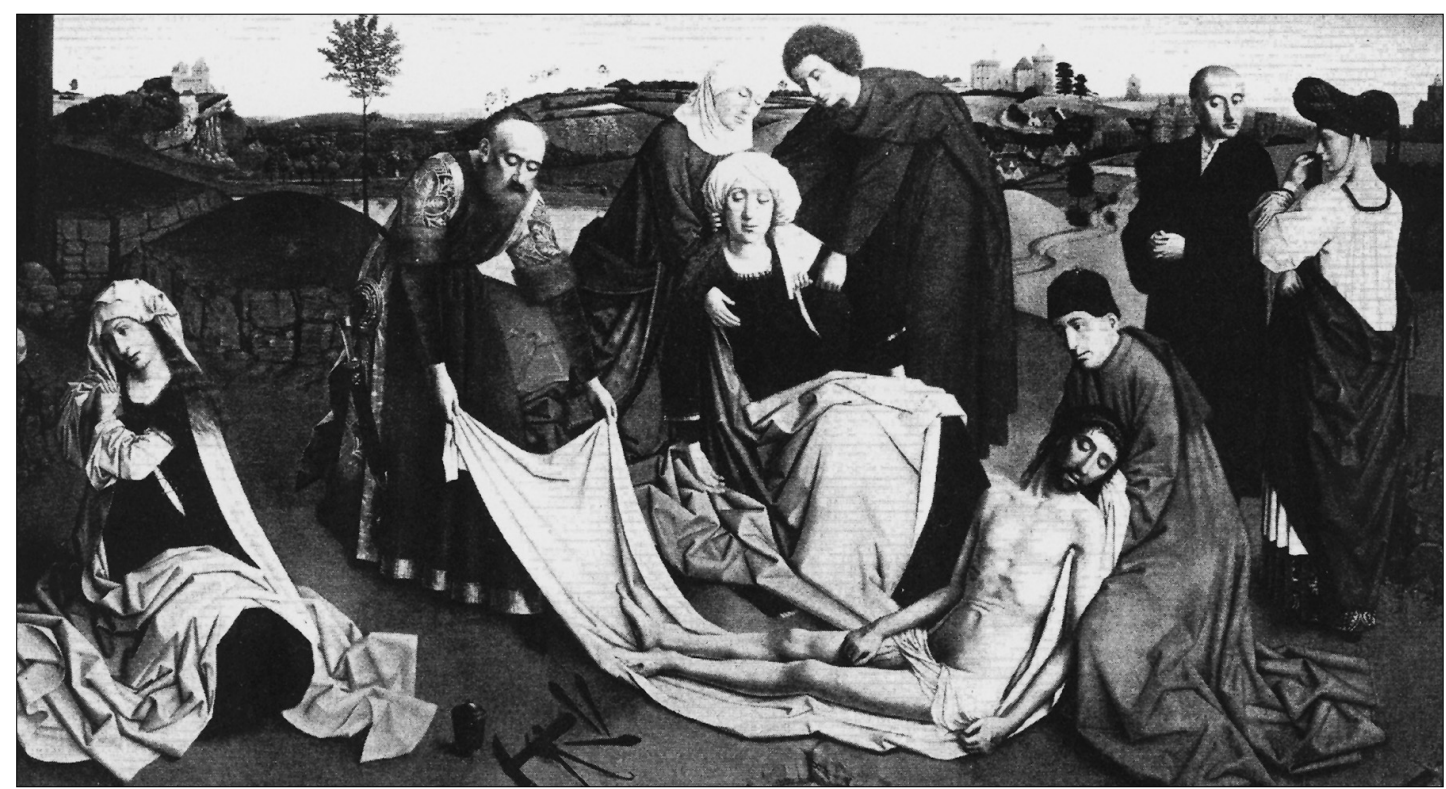

Fig. 10. Petrus Christus. La Lamentación. Bruselas. Musées Royaux des Beaux Arts. 


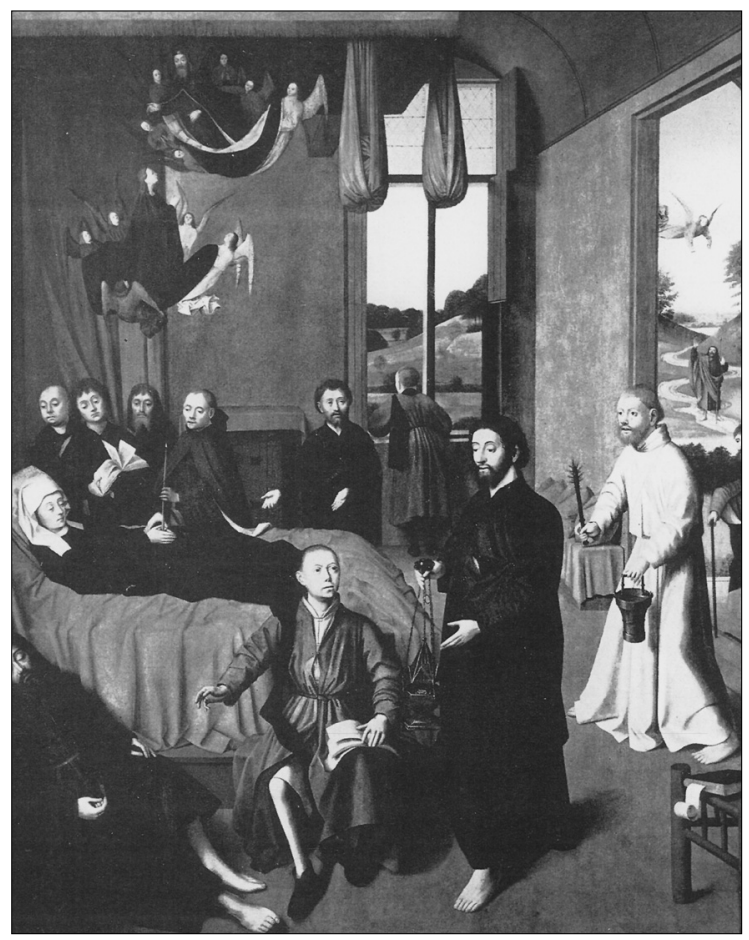

Fig. 11. Petrus Christus. La Muerte de la Virgen. San Diego. The Putnam Foundation.

Christus de hacia $1450^{11}$. Como en este cuadro de Nueva York, el cuerpo de Cristo en Avila reposa, sobre el sepulcro en este caso, formando un semicírculo en cuyos extremos se representan las figuras de José de Arimatea y Nicodemo personajes que también aparecen en la tabla de Estados Unidos aunque su tema y por lo mismo su denominación sea diferente. Sobre todo la configuración anatómica es muy similar en ambas pinturas aunque la posición de la cabeza del Cristo en la de Avila es más erguida.

También se aprecian en el tríptico de Cristo Salvador otros estilemas de la obra de Petrus Christus como pueden percibirse por ejemplo en otra versión de la Lamentación, tabla conservada en el Museo Real de Bruselas (fig. 10) que curiosamente es la misma composición, invertida, que la que aparece en la citada tabla del mismo tema de Nueva York ${ }^{12}$.

Además se han advertido recuerdos más difusos con los que aparecen en la tabla de la Muerte de la Virgen, conservada en la Fundación Putman de San Diego ${ }^{13}$, (fig. 11) una de las composiciones más difundida del pintor, pues si bien la composición difiere de la representada en el tríptico de Avila está concebida con el mismo espíritu y pueden estudiarse características similares en por ejemplo la configuración de los rostros, los efectos claroscuristas típicos de su pintura, la rica indumentaria, prolijamente representada si bien esto es genérico en la pintura flamenca de estos años.

Anterior a nuestro estudio se recuerda el de la Conservadora de Pintura del Museo Thyssen ${ }^{14}$, que recogió los datos sobre el tríptico conocidos hasta la fecha junto a un estudio iconográfico

\footnotetext{
${ }^{11}$ Friedlander, Max J. The van Eycks- Petrus Christus. Vol I Early Netherlandish Painting. Leyden, 1967, 81 y ss. Lám. P. 85.

12 Upton, J.M. "Petrus Christi”, Les Primitifs Flamands et leur temps. De Boeck-Wesmael, Belgique 1994, 363-382Lám. 372-373.

13 FrIEDLANDER, cit. Lám. 111

14 Borobia, Mar "Un Tríptico neerlandés en el Museo de Ávila" Cuadernos de Arte e Iconografía, n 5, 1990, 1-21.
} 
profundo y particularidades físico-químicas de su soporte. Es la única referencia concreta sobre esta importante pintura que ha ayudado mucho a establecer nuestras conclusiones aunque entonces no se sugirió el nombre de Petrus Christus a quien, en este breve trabajo, se atribuye el bello Tríptico de Âvila.

No se conocen las circunstancias de la llegada de esta obra a Avila pero hay que recordar la predilección de la clientela española por la pintura flamenca y las noticias que se conocen sobre encargos de los españoles que llegaban a Flandes fuesen nobles o eclesiásticos a los que posiblemente podría añadirse algún artista que como Bartolomé Bermejo tantas coincidencias presenta con la personalidad de Petrus Christus y que pudo admirar sus obras en su viaje a aquellas tierras flamencas ${ }^{15}$.

Recibido: 12-XI-2005

Aceptado: 15-V-2006

15 Bermejo, Elisa "Bartolomé Bermejo, un pintor viajero"La pintura gótica hispano-flamenca: Bartolomé Bermejo y su época Barcelona. Museu Nacional d’Art de Catalunya, 2003.

AEA, LXXX, 320, OCTUBRE-DICIEMBRE 2007, 367-377, ISSN: 0004-0428 